CONGENITAL HEART DISEASE

\title{
Current patterns of infective endocarditis in congenital heart disease
}

\author{
S Di Filippo, F Delahaye, B Semiond, M Celard, R Henaine, J Ninet, F Sassolas, A Bozio

See end of article for authors' affiliations .....................

Correspondence to: Dr Sylvie Di Filippo, Cardiologie Pediatrique, Hôpital L Pradel, 28 Avenue Doyen Lepine, 69677, Lyons, France; sylvie.di.filippo@ wanadoo.fr

Accepted 14 June 2006 Published Online First 3 July 2006

\begin{abstract}
Objective: To assess the changing profile of infective endocarditis in patients with congenital heart disease. Methods: All cases diagnosed from 1966 to 2001 (revised Duke criteria) were retrospectively reviewed and categorised in periods I $(<1990)$ and II $(\geqslant 1990)$.

Results: 153 episodes occurred, 81 in period I and 72 in period II. Mean age of affected patients was higher in period II. Non-operated ventricular septal defect, Rastelli correction and palliated cyanotic heart disease increased. Infective endocarditis in corrective surgery changed to patients with prosthetic material. Post-surgical cases decreased. Dental problems were the leading cause (period I 20\% v || 33\% of cases) with a large variety of pathological organisms (multiple species of Streptococcus). Cutaneous causative infections increased $(5 \%$ to $17 \%)$ with different species of Staphylococcus. Negative blood cultures lessened $(20 \%$ to $7 \%, p=0.03)$. Streptococci were the most common causative organisms in both periods. Severe heart failure and cardiac complications lessened $(20 \%$ to $4 \%$ and $31 \%$ to $18 \%$ during periods I and II, respectively). Early surgery was more frequent in period II $(32 \% v 18.5 \%, p=0.02)$. One- and 10 -year survival was $91 \%$ v $97 \%$ in period I and $89 \%$ v $97 \%$ in period II, respectively (NS). Conclusion: Current targets include complex cyanotic disease, congenital heart disease corrected with prosthetic material and small ventricular septal defect. Postoperative cases lessened; dental and cutaneous causes increased. Survival was unchanged. Prophylactic measures targeted at dental and cutaneous sources should be emphasised.
\end{abstract}

A ccording to a recent publication, the incidence of infective endocarditis (IE) is not decreasing. ${ }^{1}$ The relative incidence of IE in congenital heart disease (CHD) has increased as a result of improved survival with $\mathrm{CHD}^{2}$ With prolonged survival and more complex surgery, current patterns of IE in CHD may differ from previous data. Recent guidelines have redefined the at-risk groups and indications for prophylaxis. ${ }^{3}$ Some have even argued against the need for prophylaxis. ${ }^{4}$ On the other hand, microbial and imaging techniques, as well as early surgical management, may have improved outcome for patients with IE.

The objective of the present study was to review our longterm single-centre experience with IE in children and adults with CHD, and to assess the changing patterns and current profile of IE in CHD.

\section{PATIENTS AND METHODS}

The study was approved by the ethics committee of the Hospices Civils de Lyon. All episodes of IE that occurred in children and adults (age $>18$ years) with CHD from 1966 to 2001 were reviewed. Revised Duke criteria were used for the definition of diagnosis. ${ }^{5}$ All cases that met the criteria for definite IE were included. Previously normal hearts or acquired cardiac lesions were excluded. Single-institution experience was preferred to lessen centre-related disparities in the management of IE.

\section{Definitions}

Underlying cardiac disease was said to be known when previously diagnosed by echocardiography or cardiac catheterisation or unknown in patients unaware of it before IE. Some of these cases of unknown CHD were, moreover, undetermined, cardiac imaging being hindered by damage due to IE.

Complex CHD designated malformations with multiple defects or valve and vessel abnormalities (for example, single ventricle with or without transposed great vessels, or valvular atresia/stenosis).

Fever was defined as a temperature of at least $38^{\circ} \mathrm{C}$.

Bacteriological diagnosis was based on at least two blood culture samples, serological diagnosis or tissue culture positive for a micro-organism apt to cause IE, or a combination of these

IE was considered to be related to recent cardiac surgery (or perioperative) when occurring within two months postoperatively. Perioperative cases were included if they presented clinical and biological criteria for sepsis, positive blood culture, or echocardiographic or anatomical lesions.

Negative designated echocardiographic findings negative for vegetation or other new lesions suggestive of infective location.

An infection site was considered to be a potential source if the infection occurred within the month preceding symptom onset and was compatible with the causative micro-organism. Dental causes included procedures regarded as significantrisk dental work and ongoing dental lesions. ${ }^{3}$

Cardiac complications were acute pulmonary oedema, heart failure, pericarditis, haemodynamic instability and cardiogenic shock. Heart failure was considered severe if the patient was in New York Heart Association class III or IV. Surgical treatment was considered early if performed within three months after diagnosis.

Early death was defined as death occurring within three months after diagnosis. Death was considered to be IE-related if due to septic, embolic or cardiac complications or to early surgery.

Abbreviations: CHD, congenital heart disease; HACEK, Haemophilus spp, Actinobacillus actinomycetemcomitans, Cardiobacterium hominis, Eikenella corrodens and Kingella kingae; IE, infective endocarditis; PCR, polymerase chain reaction; VSD, ventricular septal defect 


\section{Prophylaxis}

This study was a long-term retrospective analysis of all the IE cases that occurred since the beginning of our CHD programme. Obviously, protocols for prophylaxis have changed over time. In our experience, prophylaxis has followed the official protocols in the time period. The threedose regimen was applied until 1990; the single-dose protocol was initiated from 1990.

Adequate prophylaxis was used to define patients who received antibiotic prophylaxis in accordance with protocols in the time period for an at-risk procedure.

\section{Time periods}

The study was subdivided into periods I (1966-1989) and II (1990-2001). The choice of 1990 as the cut off for period comparison was related to changes in diagnosis or therapeutic procedures. Newer techniques to improve microbiological diagnosis were applied (BacT/Alert FA culture bottles, polymerase chain reaction (PCR) technique, detection of micro-organisms of the HACEK group (Haemophilus spp, Actinobacillus actinomycetemcomitans, Cardiobacterium hominis, Eikenella corrodens and Kingella kingae) and detection of specific antibodies). Newer antibiotic agents were used (such as ceftriaxone or teicoplanin) and antibiotic prophylaxis was changed to the single-dose regimen.

\section{Statistics}

Quantitative variables are expressed as mean (SD) and qualitative variables as percentages. Data were analysed by Student's t test for quantitative and $\chi^{2}$ test for qualitative variables. Survival was assessed by the Kaplan-Meier method. A value of $\mathrm{p}<0.05$ was considered significant.

\section{RESULTS}

One hundred and fifty-three definite IE episodes occurred (4.2/year), 81 during period I (3.5 per year) and 72 in period II (6/year, $p=0.001)$. Age at diagnosis was $13.0(10.9)$ years and was higher in period II (16.8 (12.1) v 9.6 (8.4) years, $\mathrm{p}<0.0001)$ due to the greater proportion of adults $(40 \%$ in period II $v 9 \%$ in period I, $\mathrm{p}<0.0001)$. Sex distribution was similar in both periods (43\% women).

\section{Underlying CHD}

CHD was known in 122 patients and unknown before IE in 31. Ten of these 31 had determined abnormalities: one patent ductus arteriosus with ventricular septal defect (VSD); one double discordance (congenitally corrected transposition of the great vessels) with aortic coarctation; three bicuspid aortic valves; and five cases of mitral valve disease, including two prolapses. Twenty-one abnormalities were undetermined: two of the patients were aged 39 years, one was 35 years, one was 25 years and 17 were $<18$ years, with the following locations: six aortic, six mitral, five aortic and mitral, three tricuspid and one aortic, mitral and tricuspid.

Of the patients, 39 had corrected CHD, 35 had palliated CHD and 79 had non-operated CHD, with no difference between periods (table 1). Tetralogy of Fallot decreased between the two periods from $12 \%$ to $3 \%(p=0.03)$, and complex cyanotic CHD increased from $14 \%$ to $28 \%$ $(p=0.03)$. IE in CHD with corrective surgery occurred in the short postoperative course in period I (tetralogy of Fallot, VSD patch) but late after correction in period II (right ventricle-to-pulmonary artery conduit, prosthetic valve or pacemaker). The proportion of non-operated VSDs and of mitral or aortic native valve anomalies increased. Most palliated cases of CHD were complex.
Table 1 Underlying cardiac disease in 153 episodes of infective endocarditis during two time periods

\begin{tabular}{|c|c|c|}
\hline & $<1990$ & $\geqslant 1990$ \\
\hline \multicolumn{3}{|l|}{ Previous correction } \\
\hline VSD patch & $5(23.8 \%)$ & $1(5.5 \%)$ \\
\hline VSD patch + prosthetic & 0 & $2(11.1 \%)$ \\
\hline VSD patch + other* & $4(19.0 \%)$ & $1(5.5 \%)$ \\
\hline Rastelli conduit (1 PM) & $1(4.8 \%)$ & $5(27.8) \dagger$ \\
\hline Tetralogy of Fallotł (1 PM) & $4(19.0 \%)$ & $1(5.5) \S$ \\
\hline Coarctectomy & $1(4.8 \%)$ & $1(5.5 \%)$ \\
\hline Senning & 0 & $1(5.5 \%)$ \\
\hline Switch operation & $1(4.8 \%)$ & 0 \\
\hline ASD closure + other & $1(4.8 \%)$ & $2(11.1 \%)$ \\
\hline Prosthetic valve & $3(14.3 \%)$ & $4(22.2 \%)$ \\
\hline Pacemaker & $1(4.8 \%)$ & 0 \\
\hline Total & 21 & 18 \\
\hline \multicolumn{3}{|l|}{ Previous palliation } \\
\hline SP shunt & $12(80.0 \%)$ & $16(80.0 \%)$ \\
\hline Banding + SP shunt & $1(6.7 \%)$ & 0 \\
\hline Aortic valvulotomy & 0 & $1(5.0 \%)$ \\
\hline Aortic conduit & $1(6.7 \%)$ & $1(5.0 \%)$ \\
\hline Banding + coarctectomy & 0 & $2(10.0 \%)$ \\
\hline Coarctectomy & $1(6.7 \%)$ & 0 \\
\hline Total & 15 & 20 \\
\hline \multicolumn{3}{|l|}{ Non-operated } \\
\hline VSD & $7(15.6 \%)$ & $11(32.4 \%)$ \\
\hline VSD + left heart anomaly & $3(6.7 \%)$ & $1(2.9 \%)$ \\
\hline VSD + right heart anomaly & $1(2.2 \%)$ & 0 \\
\hline PDA & $1(2.2 \%)$ & 0 \\
\hline PDA + other & $1(2.2 \%)$ & $1(2.9 \%)$ \\
\hline AVSD & $2(4.4 \%)$ & 0 \\
\hline Tetralogy of Fallot & $2(4.4 \%)$ & 0 \\
\hline MVP & $3(6.7 \%)$ & $1(2.9 \%)$ \\
\hline Mitral regurgitation & $3(6.7 \%)$ & $5(14.7 \%)$ \\
\hline Bicuspid aortic valve & $1(2.2 \%)$ & $3(8.8 \%)$ \\
\hline Aortic valve stenosis & $1(2.2 \%)$ & 0 \\
\hline Mitroaortic anomalies & 0 & $1(2.9 \%)$ \\
\hline Pulmonary valve stenosis & $1(2.2 \%)$ & 0 \\
\hline$A S D+M V P$ & $1(2.2 \%)$ & 0 \\
\hline Aortic coarctation & $1(2.2 \%)$ & 0 \\
\hline Complex CHD & $4(8.9 \%)$ & $3(8.8 \%)$ \\
\hline Undetermined & $13(28.9 \%)$ & $8(23.5 \%)$ \\
\hline Total & 45 & 34 \\
\hline \multicolumn{3}{|c|}{$\begin{array}{l}\text { *Other non-prosthetic lesion; †one Fallot with pulmonary atresia; } \\
\text { tregular Fallot; §Fallot repair with pacemaker; } \text { Tincluding four } \\
\text { tetralogies of Fallot. } \\
\text { ASD, atrial septal defect; AVSD, atrioventricular septal defect; CHD, } \\
\text { congenital heart disease; MVP, mitral valve prolapse; PDA, patent ductus } \\
\text { arteriosus; PM, pacemaker; SP, systemic-to-pulmonary; VSD, ventricular } \\
\text { septal defect. }\end{array}$} \\
\hline
\end{tabular}

\section{Sources and micro-organisms}

A source of infection was identified in $67.3 \%$ of cases: $72.2 \%$ in period II and $63 \%$ in period I (NS). Dental procedures and dental infection were more frequent in period II (33\% v 20\%, $\mathrm{p}=0.05)$. Ongoing dental infection was diagnosed after IE occurred in 24 of the 40 dental-related cases: 16 of the 40 patients had a previously recognised dental event (10 procedures and six focal infections). Prophylaxis was adequate in eight procedures, and eight patients (during two procedures) had not received prophylaxis, despite the known risk.

The rate of cutaneous infection (16 cases) rose to $17 \%$ in period II, up from $5 \%(\mathrm{p}=0.05)$, comprising minor periungual lesions or other cutaneous wounds; two of these patients had received antibiotics and 14 had not.

Perioperative infection ( 25 cases) tended to be less frequent in period II (eight perioperative cases of 72 infections $(11 \%) v 17$ of $81(21 \%)$ in period I), although non-significantly. These cases occurred after palliation of cyanotic CHD (eight cases: five in period I and three in II), after corrective surgery (VSD closure, Rastelli operation, mechanical valve replacement or Fallot correction, 16 cases: 11 in period I and five in II) or after pacemaker implantation (one case in period I). The incidences of other sources of 


\begin{tabular}{|c|c|c|c|c|c|c|c|}
\hline & Cutaneous & Dental & ENT & Surgery & Other & Unknown & Tota \\
\hline \multicolumn{8}{|l|}{ Streptococci } \\
\hline Streptococcus spp & 1 & 9 & 0 & 1 & 0 & 7 & 18 \\
\hline $\begin{array}{l}\text { adjacens (Abiotrophia } \\
\text { adjacens) }\end{array}$ & 0 & 2 & 1 & 1 & 0 & 1 & 5 \\
\hline constellatus & 0 & 1 & 0 & 0 & 0 & 0 & 1 \\
\hline gordonii & 0 & 1 & 0 & 0 & 0 & 0 & 1 \\
\hline intermedius & 0 & 1 & 0 & 0 & 0 & 1 & 2 \\
\hline mitis & 0 & 4 & 1 & 1 & 0 & 0 & 6 \\
\hline mutans & 0 & 1 & 0 & 0 & 0 & 1 & 2 \\
\hline oralis & 0 & 7 & 0 & 1 & 0 & 0 & 8 \\
\hline sanguis & 0 & 7 & 1 & 0 & 0 & 7 & 15 \\
\hline pneumoniae & 0 & 0 & 3 & 0 & $1^{*}$ & 1 & 5 \\
\hline \multicolumn{8}{|l|}{ Enterococci } \\
\hline \multicolumn{8}{|l|}{ Staphylococci } \\
\hline aureus & 15 & 1 & 1 & $12 \ddagger$ & $1^{*}$ & 9 & 39 \\
\hline Coagulase negative & 0 & 0 & 0 & 1 & 0 & 4 & 5 \\
\hline \multirow{2}{*}{\multicolumn{8}{|c|}{ Gram-negative bacilli }} \\
\hline & & & & & & & \\
\hline Acinetobacter spp & 0 & 1 & 0 & 0 & 0 & 0 & 1 \\
\hline Escherichia coli & 0 & 0 & 0 & 3 & $1 \S$ & 1 & 5 \\
\hline Klebsiella oxytoca & 0 & 0 & 0 & 1 & 0 & 0 & 1 \\
\hline Pseudomonas aeruginosa & 0 & 0 & 0 & 2 & 0 & 0 & 2 \\
\hline Brucella melitensis & 0 & 0 & 0 & 0 & 0 & 1 & 1 \\
\hline Kingella kingae & 0 & 0 & 0 & 0 & $1 \dagger$ & 0 & 1 \\
\hline \multicolumn{8}{|l|}{ Gram-positive bacilli } \\
\hline Lactobacillus spp & 0 & 0 & 0 & 0 & 0 & 1 & 1 \\
\hline Propionibacterium acnes & 0 & 0 & 0 & 0 & 0 & 3 & 3 \\
\hline \multicolumn{8}{|l|}{ Others } \\
\hline Mycoplasma pneumoniae & 0 & 0 & 0 & 0 & 0 & 1 & 1 \\
\hline Coxiella burnetii & 0 & 0 & 0 & 0 & 0 & 1 & 1 \\
\hline Aspergillus spp & 0 & 0 & 0 & 0 & 0 & 1 & 1 \\
\hline Unknown & 0 & 4 & 2 & 3 & 5 & 7 & 21 \\
\hline Total & 16 & 40 & 10 & 27 & 10 & 50 & 153 \\
\hline
\end{tabular}

infection were similar in both periods (ear, nose and throat, 10 cases; digestive, six cases; and pulmonary or urological, six cases).

Table 2 lists the causative agents and sources of infection. The proportion of negative blood cultures decreased from $20 \%$ to $7 \%(p=0.03)$ (fig 1$)$. Streptococci were the most common agents; their frequency varied from $31 \%$ to $47 \%$ of all cases and $41 \%$ to $55 \%$ of all documented micro-organisms between periods I and II (NS). Staphylococcus aureus and coagulase-negative staphylococci rates did not change $(26 \%$ and $25 \%$, and $5 \%$ and $4 \%$, respectively). Death and early surgery rates were similar between nosocomial staphylococci (14 cases) and staphylococci of other origins. Other microorganisms, such as Propionibacterium acnes (three cases), emerged in period II.

\section{Clinical data}

Mean time from symptom onset to diagnosis was 34 days in period II and 41 days in period I (NS). Prolonged fever was the most frequent symptom (99\%). The rate of severe heart failure decreased from $20 \%$ to $4 \%(p=0.005)$ as did that of cardiac complications, from $31 \%$ to $18 \%(p=0.07)$. An embolic event occurred in $35 \%$ of cases, with no difference between periods.

\section{Location of infection and echocardiographic lesions}

Echocardiographic imaging records (transthoracic or transoesophageal) were retrieved in 134 cases (88\%). Forty-nine echocardiographic records (36.5\% of all echographic records) were negative. Echocardiographic studies were negative in $70 \%$ of cases of IE occurring in palliated complex CHD. Vegetations were the most frequent lesions (46\%). An echographic complication was diagnosed in 21 cases $(16 \%)$ : eight valve perforations, five chordae tendineae ruptures, three cases of prosthesis, one annular abscess, one VSD and one aortic aneurysm.

Locations were similar in both periods. Thirty were in the right heart (four VSD; four right ventricle free wall; 17 tricuspid or pulmonary valve; two pulmonary artery; one pacemaker; and two right ventricle to pulmonary artery conduit), 60 were in the left heart (18 native aortic valve; 28 native mitral valves; nine both mitral and aortic valves; two aortic prosthetic valves; and three aorta), five were in both the left and right heart and three were on systemic-topulmonary shunts.

\section{Surgical management}

Surgery was early in 38 cases $(24.8 \%)$, including for 13 streptococcal IEs (for a total of 64 streptococcal IEs; $20 \%$ ) and 14 staphylococcal IEs (30\% of 46). Indications for early surgery were haemodynamic impairment for $50 \%$ of operated cases, iterative embolism for $14 \%$, failure to control sepsis for $3 \%$, or other for $33 \%$ (pacemaker removal or annular abscess).

Surgery was early in 15 period I cases (18.5\%) and 23 period II cases $(31.9 \%, p=0.02)$. The valvular procedures ( 23 of 38 early surgical interventions) in period I were eight prostheses, three aortic homografts and one mitral plasty; in period II they were five prostheses, four Ross procedures and two mitral plasties. Additional procedures in period I were one aortoaortic tube, one pacemaker removal and one ductus arteriosus ligation; in period II they were one Rastelli tube change, one aortoaortic tube, two VSD patches, two pacemaker removals, two aneurysm resections, two pericardiocenteses and one systemic-to-pulmonary shunt. 


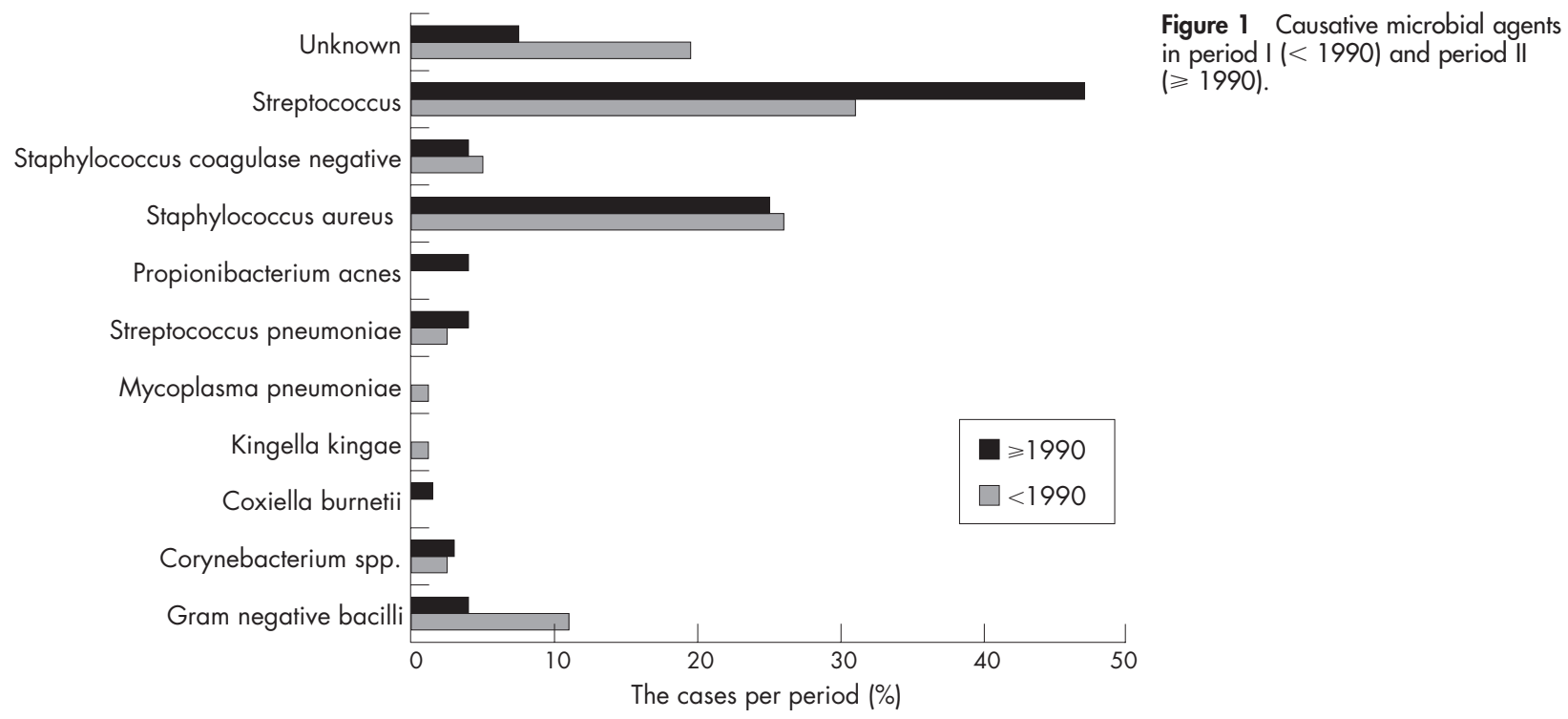

\section{Survival}

Mean follow up was 6.1 (6.6) years ( 3 days to 26.9 years): 9.9 (7.5) for period I and 2.6 (2.8) years for period II. In-hospital mortality was $7.2 \%$ ( 11 cases): $8.6 \%$ in period I (seven cases due to cardiac, embolic or septic complications) and $5.6 \%$ in period II (four cases, postoperatively, NS). Survival was $91 \%$ and $90 \%$ at one year, and $79 \%$ and $90 \%$ at 10 years, respectively, in periods I and II (NS). The rate of freedom from IE-related mortality was $91 \%$ and $97 \%$ at one year, and $89 \%$ and $97 \%$ at 10 years, respectively, in periods I and II (NS).

\section{DISCUSSION}

In some institutions, such as our own, the proportion of CHD in adults with IE is high, probably as a result of patient selection; in others, CHD accounted for only $1 \%$ of cases. ${ }^{6}$ As more patients with even complex CHD are reaching adulthood, the number of cases of IE in adults with CHD has increased. ${ }^{2}$ Lack of information given to patients and noncompliance with prophylaxis may also account for the rising frequency of IE in adults with CHD. Moons et $a l^{7}$ found that only $48 \%$ of adults with CHD were clearly aware of their cardiac disease and only 16\% knew about the risk of IE and about prophylaxis. Our findings raise the question of whether follow up and education should be continued for patients with CHD reaching adulthood. IE should probably be one of the major educational issues for specific adult CHD programmes.

Because they have multiple cardiac lesions such as intracardiac defects and valve regurgitation or stenosis, patients with complex cyanotic CHD carry the highest risk for IE. ${ }^{8-11}$ Although palliative surgical procedures prolong survival, they have created additional targets for infection (for example, shunts). The rate of complex palliated CHD in IE may reach $35 \%$ of cases $^{812} 13$ and our results are in accordance with these assessments.

Surgical repair can eliminate the risk of IE if no residual lesion is present. ${ }^{214} 15$ For example, tetralogy of Fallot has become a less common IE target. About $75 \%$ of our patchclosed VSD cases in period I and $100 \%$ in period II occurred in the early postoperative course; none occurred late $(>6$ months) after surgery if no residual shunt or valvular lesion was left. However, corrective surgery is not always protective and may itself create potential targets for infection, such as conduits or prostheses. ${ }^{214}$ The Rastelli right ventricle-to-pulmonary artery conduit was one such new target in our experience.

A high prevalence of small VSDs, enhanced diagnostic ability, and lack of cardiac surveillance and education may combine to account for the increasing frequency of nonoperated VSD between periods I and II. The risk may have previously been underestimated in these asymptomatic patients whose cardiac lesion was considered benign. Of note, guidelines have classified VSD as moderate-risk lesions. ${ }^{3}$ We would not recommend closing all small VSDs but would encourage caregivers not to underestimate the infectious risk in this group of patients. Although mitral valve prolapse has been implicated in a rising number of cases in adults, our study did not confirm this assessment. ${ }^{16}$ However, mitral valve prolapse could have been present in six patients with undetermined underlying cardiac disease and mitral valve IE. Similarly, aortic bicuspid valves probably are a nonnegligible target for IE. ${ }^{17}$

Dental events were still the most common and even increasing causal factor. The reported incidence of dental IE varies from $11 \%$ to $40 \%$, depending on whether invasive procedures or poor dental hygiene is taken into consideration. $^{18}{ }^{19}$ Because of our growing cohort of patients, we assume that the number of patients undergoing regular dental care has increased over the years. That may account for the paradoxically rising number of dental-related IE in the present study. Ten of our IE cases were related to recent dental work: eight patients had received adequate prophylaxis. The total number of protected dental procedures performed during the same period was not known, but this nevertheless confirms that prophylaxis is not always protective. Whether all dental procedures need prophylaxis remains a subject of debate. ${ }^{40} \mathrm{~A}$ recent study showed that the frequency of positive blood culture was significantly lower in randomly selected patients who had received prophylaxis for a dental procedure than in those who had not. ${ }^{21}$ We therefore intend to follow the official guidelines for theoretically at-risk events. ${ }^{32}$ Not only dental procedures but also unrecognised dental and gingival lesions are potential sources of infection. Poor dental hygiene probably presents the highest risk, likely to be underestimated by both patients and physicians; ongoing dental infections were diagnosed only after IE had occurred in $60 \%$ of our dental cases. As mentioned by Roberts et al, ${ }^{20}$ daily brushing or gum chewing may be unpredictable events that can hardly be prevented. These events might have 
contributed to streptococcus-related IE with an unrecognised source of infection.

In our experience, more attention should be paid to cutaneous sources of infection. Cutaneous infections were all misdiagnosed. Although prophylaxis protocols usually do not include cutaneous risk, dermatologists have established guidelines for prophylaxis in high-risk patients undergoing dermatological procedures. ${ }^{23}$ Daily hygiene probably is the most crucial issue to be addressed. Ongoing minor skin lesions emerged as increasingly frequent sources of IE. Some of these may have been overlooked in the past, leading to $S$ aureus IE.

Newer techniques to aid conventional microbiological diagnosis probably contributed to decreased rates of culture-negative IE. Other studies have reported a $9 \%$ incidence of negative blood culture, concordant with our present results. ${ }^{2}$ Of high interest is the PCR technique recently used to detect microbial nucleic acids in heart valves in patients with IE, by means of culture-independent, broad-range amplification methods. ${ }^{24}$ Six conserved areas in 16S rRNA genes of bacteria (1600 bp) are suitable for use as panbacterial primer targets. Several fastidious, endocarditiscausing pathogens such as those in the HACEK group, Coxiella burnetii, Legionella pneumophila, Chlamydia, Mycoplasma, Brucella, Bartonella, Ehrlichia and Tropheryma whippelii can be detected in a single assay. Being able to detect genes in staphylococci and streptococci at the species level (speciesspecific PCR) is also advantageous. However, DNA from the causative agent(s) must be present at a high concentration to be detected. Sensitivity achieved by broad-range PCR strategies for the more common pathogenic agents of IE often is no better than that with blood culture. This type of analysis would be the most relevant in precise clinical cases of culture-negative IE. Moreover, the diagnosis by heart valve analysis is not helpful in guiding initial treatment, except in patients who undergo early emergency surgery. Streptococci were the most common micro-organisms in our experience and paralleled dental sources as persistent major causes of IE. Staphylococcus was most frequent in some other reports but was associated with central venous catheters in patients with normal hearts, who were excluded from the present study. ${ }^{10}{ }^{11}$ Staphylococcus was our second most frequent microbial agent, despite a falling rate of perioperative infection, probably as a result of increasing cutaneous sources.

Echocardiography is one major criterion for diagnosis of IE. ${ }^{525-27}$ No reports have assessed the sensitivity of echocardiography in the diagnosis of IE in the specific group of patients with complex cardiac malformations. Echocardiographic imaging was negative in $70 \%$ of our complex CHD cases. Difficulty in imaging shunts and conduits and in differentiating vegetation from dysplastic exuberant valves combine to weaken the sensitivity of echocardiography in the group of patients with complex CHD. Careful comparison of valve anatomy and function with previous records is required to detect relevant differences. Transoesophageal echocardiography may help to acquire prognostic information or to assess IE-related complications, and may be more sensitive than transthoracic echocardiography in patients with poor echogenicity, ${ }^{28}$ but it also fails to image the lesion in patients with complex cardiac anatomy. Diagnosis of IE should therefore rely on Duke criteria other than echocardiography in complex cardiac lesions, conduits and shunts.

Overall mortality decreased, although not significantly. Early mortality was $7.2 \%$ in the present study and varies from $10-25 \%$ in the literature. ${ }^{8-11}$ Improvement in early survival may be a consequence of earlier and more efficient surgical treatment as reported in the literature. ${ }^{29}$ However, improved microbiological techniques and diagnosis, as well as more specific and stronger antibiotic treatment, may also account for the lower number of early deaths.

\section{Study limitations}

There are several limitations to the present study. Firstly, the retrospective nature of the analysis did not allow for control of data and requires cautious interpretation of the results.

Some cases that occurred in the very beginning of our experience may have been missed.

Focal infections that occurred in the month preceding symptom onset were considered to be potential sources of IE, if compatible with the causative microbial agent. If blood culture was negative, only ongoing focal infection was taken into account. Instead, some authors would regard two weeks as a reasonable interval between source of infection and symptom onset, although no reports have clearly defined this incubation period; it probably depends on the virulence of the causative agent as well as the underlying predisposing cardiac disease.

This study is an overview of the current and changing patterns of IE in CHD. Several points were highlighted (increasing proportion of adults, small VSD as an underestimated target, cutaneous source of infection and negativity of echocardiographic findings) that would have to be specifically addressed in further analyses.

\section{Conclusion}

Our experience with IE in patients with CHD showed an increased proportion of adult cases. Current targets for IE include unrepaired complex cyanotic CHD, CHD corrected with prosthetic material and small VSDs. Dental causes were still predominant and cutaneous causes increased. Despite significant improvement in bacteriological diagnosis and more frequent surgical management, early mortality did not change significantly over time. In the light of these results, prophylactic measures targeted at dental and cutaneous sources should be emphasised.

\section{Authors' affiliations}

S Di Filippo, B Semiond, F Sassolas, A Bozio, Cardiologie Pediatrique, Hopital L Pradel, Lyons, France

F Delahaye, Cardiologie A, Hopital L Pradel, Lyons, France

M Celard, Microbiologie-Bacteriologie, Hôpital L Pradel, Lyons, France R Henaine, J Ninet, Chirurgie Cardiothoracique, Hopital L Pradel, Lyons, France

\section{REFERENCES}

1 Niwa K, Nakazawa M, Tateno S, et al. Infective endocarditis in congenital heart disease: Japanese national collaboration study. Heart 2005;91:795-800

2 Li W, Somerville J. Infective endocarditis in the grown-up congenital (GUCH) population. Eur Heart J 1998;19:166-73.

3 Horstkotte D, Follath F, Gutschik E, et al. Guidelines on prevention, diagnosis and treatment of infective endocarditis. The task force on infective endocarditis of the European Society of Cardiology. Eur Heart J 2004;25:267-76.

4 Strom BL, Abrutyn E, Berlin JA, et al. Dental and cardiac risk factors for infective endocarditis: a population-based, case-control study. Ann Intern Med 1998; 129:761-9.

5 Durack DT, Lukes AS, Bright DK. New criteria for diagnosis of infective endocarditis: utilization of specific echocardiographic findings. Am J Med 1994;96:200-9.

6 Hoen B, Alla F, Selton-Suty C, et al. Changing profile of infective endocarditis: results of a 1-year survey in France. JAMA 2002;288:75-81.

7 Moons P, De Volder E, Budts W, et al. What do adult patients with congenital heart disease know about their disease, treatment, and prevention of complications? A call for structured patient education. Heart 2001;86:74-80.

8 Fisher MC. Changing risk factors for pediatric infective endocarditis. Curr Infect Dis Rep 2001;3:333-6.

9 Dodo H, Child JS. Infective endocarditis in congenital heart disease. Cardiol Clin 1996;14:589-602.

10 Moller JH, Anderson RC. 1,000 consecutive children with cardiac malformation with 26- to 37-year follow-up. Am J Cardiol 1992;70:661-7.

11 Martin JM, Neches WH, Wald ER. Infective endocarditis: 35 years of experience at a children's hospital. Clin Infect Dis 1997;24:669-75. 
12 Geva T, Frand M. Infective endocarditis in children with congenital heart disease: the changing spectrum 1965-85. Eur Heart J 1988;9:1244-9.

13 Awadallah SM, Kavey REW, Byrum CJ, et al. The changing spectrum of infective endocarditis in childhood. Am J Cardiol 1991;68:90-4.

14 Morris CD, Reller MD, Menashe VD. Thirty-year incidence of infective endocarditis after surgery for congenital heart defect. JAMA 1998; 179:599-603

15 Karl T, Wensley D, Stark J, et al. Infective endocarditis in children with congenital heart disease: comparison of selected features in patients with surgical correction or palliation and those without. Br Heart J 1987;58:57-65.

16 Michel PL, Acar J. Native cardiac disease predisposing to infective endocarditis. Eur Heart J 1995; 16:2-6.

17 Yener N, Oktar GL, Erer D, et al. Bicuspid aortic valve. Ann Thorac Cardiovasc Surg 2002;8:264-7.

18 Raymond J, Aujard Y, the European Study Group. Nosocomial infections in pediatric patients: a European, multicenter prospective study. Infect Control Hosp Epidemiol 2000;21:260-3.

19 Al-Karaawi ZM, Lucas VS, Gelbier M, et al. Dental procedures in children with severe congenital heart disease: a theoretical analysis of prophylaxis and non-prophylaxis procedures. Heart 2001;85:65-8.

20 Roberts GJ. Dentists are innocent! "Everyday" bacteremia is the real culprit: a review and assessment of the evidence that dental surgical procedures are a principal cause of bacterial endocarditis in children, Pediatr Cardiol 1999:20:317-25.

21 Lockhart PB, Brennan MT, Kent ML, et al. Impact of amoxicillin prophylaxis on the incidence, nature, and duration of bacteremia in children after intubation and dental procedures. Circulation 2004; 109:2878-84.
22 Dajani AS, Taubert KA, Wilson W, et al. Prevention of bacterial endocarditis. Recommendations by the American Heart Association. Circulation 1997:96:358-66.

23 Conway DSG, Taylor AD, Burrell CJ. Atopic eczema and staphylococcal endocarditis: time to recognize an association? Hosp Med 2000;61:356-7.

24 Grijalva M, Horvath R, Dendis $M$, et al. Molecular diagnosis of culture negative infective endocarditis: clinical validation in a group of surgically treated patients. Heart 2003;89:263-8

25 Greaves K, Mou D, Patel A, et al. Clinical criteria and the appropriate use of transthoracic echocardiography for the exclusion of infective endocarditis. Heart 2003;89:273-5.

26 Gomez-Nunez N, Vargas-Barron J, Espinola-Zavaleta N, et al. Echographic study of patients with congenital heart disease and infective endocarditis. Echocardiography 2001;18:485-90.

27 Aly AM, Simpson PM, Humes RA. The role of transthoracic echocardiography in the diagnosis of infective endocarditis in children. Arch Pediatr Adolesc Med 1999;153:950-3.

28 Thangaroopan M, Choy JB. Is transesophageal echocardiography overused in the diagnosis of infective endocarditis? Am J Cardiol 2005;95:295-7.

29 Picarelli $\mathrm{D}$, Leone R, Duhagon $\mathrm{P}$, et al. Active infective endocarditis in infants and childhood: ten-year review of surgical therapy. J Card Surg 1997; 12:406-11.

30 Ferrieri P, Gewitz MH, Gerber MA, et al. Unique features of infective endocarditis in childhood. Circulation 2002;105:21 15-27.

\section{IMAGES IN CARDIOLOGY}

\section{Pseudoaneurysm of aortocoronary graft secondary to spontaneous late rupture}

A

79-year-old man with a prior history of three-vessel coronary artery bypass graft surgery performed 14 years ago was admitted for chest pain radiating into the back. Chest $x$-ray and spiral computed tomographic scan (panels A and B) were performed and showed a $6.7 \times 5.1 \times 5.3 \mathrm{~cm}$ mass in the left hilum, likely representing a pseudoaneurysm with an active leak from an adjacent bypass graft. Emergency coronary angiography found a pulsatile extravasation of contrast product superimposed on the soft tissue mass of the pseudoaneurysm in the never instrumented mid portion of the vein graft to the left anterior descending coronary artery (panel C). The localised spontaneous rupture of the graft was successfully treated by implanting a covered JoStent (panel D). The stenotic distal anastomosis was also successfully treated with a Cypher drug eluting stent. The patient was discharged without any other issue.

E Flecher

S Wilson

C M G Duran

erwan.flecher@wanadoo.fr
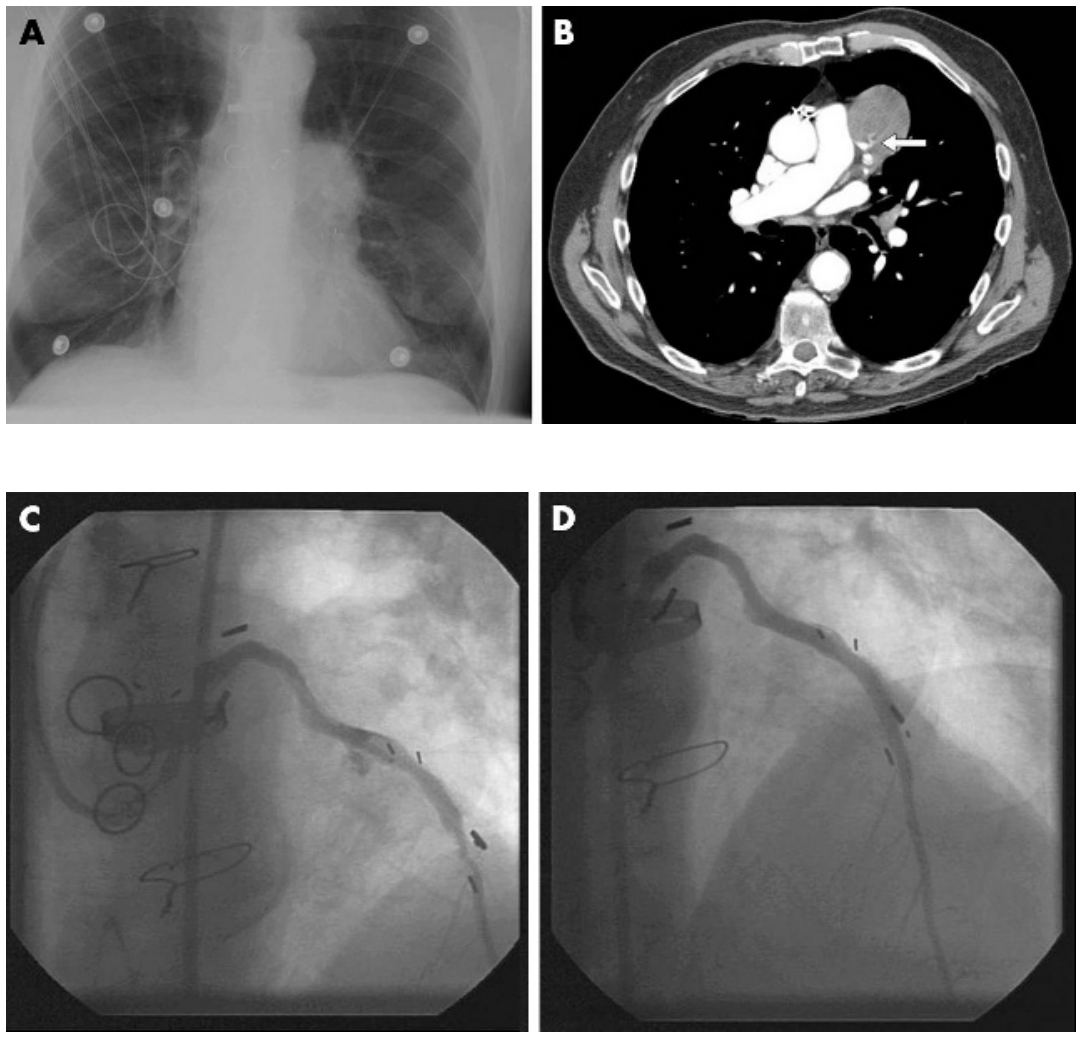